\title{
Complex Temporal Dynamics in Optical Cavities
}

\author{
T. Hansson \\ Department of Applied Physics \\ Chalmers University of Technology \\ Göteborg, Sweden \\ tobhan@chalmers.se
}

\author{
D. Modotto, S. Wabnitz \\ Dipartimento di Ingegneria dell'Informazione \\ Università di Brescia \\ Brescia, Italy \\ stefan.wabnitz@unibs.it
}

\begin{abstract}
We overview recent advances in the modeling and understanding of complex spatiotemporal dynamics associated with the generation of optical frequency combs in microresonators.
\end{abstract}

Keywords-resonators; four-wave mixing; optical solitons; instabilities; integrated optics

\section{INTRODUCTION}

The spatiotemporal dynamics of microresonator frequency comb generation is currently a hot research topic, which is being investigated both experimentally and theoretically by several groups. Laser frequency comb sources have revolutionized precision frequency metrology as recognized by the Nobel Prize in 2005. Applications for frequency combs include, e.g., spectroscopy, optical clocks, waveform synthesis, wavelength-division multiplexed telecommunication sources and astronomical spectrograph calibration. Frequency comb sources based on microresonators offer an intriguing alternative to mode-locked lasers with several unique benefits [1]. From a fundamental point of view, microresonators have been found to exhibit a rich dynamics, including stationary patterns and cavity solitons as well as chaotic states.

Commonly investigated microresonators are planar microrings and crystalline whispering gallery mode resonators. Among these, planar resonators enjoy several benefits. Indeed, they can be fabricated with low cost on a chip to form integrated photonics circuits, thus having potential for mass manufacturing. In contrast to crystalline microresonators, microrings do not require mechanical polishing, and can be made in different shapes, such as racetracks. On the other hand, the main drawback of planar resonators is that they have a lower quality (Q) factor when compared to crystalline resonators. Suitable materials should be chosen according to the desired emission wavelength range, as determined by their transparency window (absorption, two-photon absorption (TPA), free-carrier absorption (FCA)) and dispersion profile, which however can be engineered by using the waveguide geometry.

In this work we overview the theory of microresonator frequency comb generation and discuss some recent advances and applications. We begin by providing the fundamentals of modeling, which involve the spatial problem and the different approaches to treat the temporal dynamics. These include the

This work was sponsored by the Italian Ministry of the University and Research and by the Swedish Research Council.
Ikeda map [2], the coupled wave approach [3], and the mean field approach [4][5], which leads to the Lugiato-Lefever equation (LLE) [6]. We discuss the physical mechanisms of frequency comb generation, based on modulation instability (MI) and cascade four-wave mixing (FWM) [7][8][9]. Modeling of the time dynamics may exploit truncated models, which permit analytical studies of the comb stability [9]. This simplified approach may also be used in the case of a bichromatically pumped comb, which leads to thresholdless comb generation [10]. We further introduce the concept of temporal cavity soliton, which results from a dissipative balance between cavity loss and periodic pump injection [11]. Only recently observed in passive fiber cavities [12], temporal cavity solitons are associated with stable phase-locked comb states in microresonators [13].

We consider next the high power regime, where the LLE fails to reproduce the richness of the temporal dynamics, which exhibits for example period-2 (P2) instabilities [14]. A remarkable property of the high-power regime is the coexistence of multistable solitons or super cavity solitons [15].

In terms of applications, we focus our attention to the possibility of obtaining wideband mid-infrared (MIR) comb generation in silicon microresonators [16]. We introduce a suitable nonlinear envelope equation for comb dynamics in semiconductor microresonators, and show that multiphoton absorption leads to free-carrier dispersion induced cavity soliton generation even for initial zero cavity detuning of the pump beam [17]. We conclude by outlining the possibility of observing Raman frequency combs in silicon microresonators.

\section{FUNDAMENTALS OF MODELLING}

Microresonators are fundamentally described by the wave equation for the electric field, which can be separated into spatial and temporal parts. The spatial part determines the resonant modes along with their eigenfrequencies, and the associated dispersion properties [18]. The dynamics is determined by the temporal part, and uses the solution of the spatial part as input for the parameters in the evolution equations. The spatial problem is typically solved for the resonant eigenmodes and eigenfrequencies by using a numerical mode solver (e.g., COMSOL). The cavity dispersion depends on the nonequidistance of the resonator mode frequencies. Various analytical approaches have also been developed that allow for approximating the dispersion 
properties, and describe e.g. the TE/TM mode families of planar microring resonators [19].

As far as the time dynamics is concerned, two main models are used for describing frequency comb generation in microresonators, namely the driven and damped nonlinear Schrödinger (NLS) equation[4][5][9], also known as the LLE [6], and the coupled mode equations [3]. Both models are equivalent, and provide complementary views on the comb generation process. Basically, the driven and damped NLS is a time-domain description, while the coupled mode model is a frequency-domain description. Both models can be efficiently simulated using split-step Fourier methods: the choice of model depends on convenience for any particular problem. Indeed, it has been shown that fast Fourier transform techniques can be used to calculate the nonlinear term in the coupled mode equations, which allows for octave spanning combs to be efficiently simulated, with a typical simulation time for a 4096 mode comb of about 1 minute on a desktop computer [20].

A more general model to describe the time dynamics of microresonator frequency combs is provided by the Ikeda map [2], which uses the NLS equation for propagating the electric field envelope in the resonator waveguide, coupled with the cavity boundary conditions relating fields between each roundtrip. This approach should be used whenever the linear (due to pump detuning from resonance) or the nonlinear (due to the cumulated over the cavity intensity dependent index) phase shifts are not small when compared to unity. Again, the Ikeda map can be easily numerically simulated using the standard split-step Fourier method. However in this case the integration step should be much smaller than the cavity length.

The LLE is obtained, if both detuning and nonlinear phaseshift are small, as the mean-field limit of the Ikeda map, i.e., by averaging the map over one round-trip (integration over space) [7][8]. In this way, two time-scales are introduced: namely, ordinary (fast) time and a (slow) evolution time variable. In contrast to the case of passive fiber cavities, in microresonator the generated temporal structures can have a time duration that is comparable to the cavity round-trip time: this requires the use of periodic boundary conditions in the fast time variable, which means that the resulting LLE has three (as opposed to two for the fiber cavity case) independent free parameters [9].

\section{MECHANISMS OF FREQUENCY COMB GENERATION}

Frequency comb generation occurs principally owing to MI of the bistable pump mode. The MI process creates the primary sidebands, which interact with the pump mode to create additional sidebands through FWM. The sidebands can, depending on external pump intensity and detuning, become sufficiently strong so that they may also experience MI, which creates subcombs. The comb formation process is also aided by cascaded FWM, which may create new sidebands even further away from the pump, and may produce ultra wideband combs that span an entire octave. MI is analyzed by means of a Fourier expansion (equivalent to the coupled mode equations) and linearizing around the continuous wave (CW) solution [9]. The pump mode of a CW driven cavity has either one or three steady-state solutions, thus it exhibits bistability if the cavity detuning is large enough: the negative slope branch of the bistable cavity transmission is unstable with respect to $\mathrm{CW}$ perturbations. By considering the growth rate of two cavity modes (symmetric with respect to the $\mathrm{CW}$ mode) with respect to the growth of periodic modulations, one obtains that instability of a given pump mode is obtained whenever its amplitude exceeds a certain threshold value (in contrast to MI of the CW solution of the NLS equation, where no threshold exists). Physically, this is due to the fact that parametric gain must overcome the cavity loss for the instability to develop. It is remarkable however that cavity MI can, due to the detuning, occur both in the normal and in the anomalous dispersion regime; moreover, sidebands close to the pump frequency are not amplified.

Insight into the dynamics of frequency comb generation can readily be obtained using finite mode truncations [9]. The rationale for this approach is that a substantial part of the total energy is contained in the pump mode and a small number of sidebands. The main advantage of a truncated model is that it is much simpler than solving the full LLE, as the problem is reduced to solving a finite number of ordinary differential equations (ODEs). Moreover the model describes pump depletion hence it goes beyond the linearized MI analysis. A mode truncation replicates the dynamics within a low dimensional system, which allows the location of different solution branches to be found, and it may permit to analytically predict regimes when comb generation is unstable. On the other hand, by definition a finite mode truncation neglects additional sidebands, hence it cannot be used to predict absolute comb stability. Moreover, the resulting pump mode and sideband amplitudes are not always accurate.

Nevertheless, a three-mode truncation permits to identify both soft (i.e., induced by MI of the pump mode) and hard (that is, not directly accessible from the pump mode via the MI process activated by noise) frequency combs, in particular in the normal dispersion regime of the cavity. Comb states have also been found to appear for specific parameter values in the hard excitation region of the normal dispersion regime: however these combs typically have a finite lifetime. It is also interesting to note that, in addition to the steady-state $\mathrm{CW}$, also frequency combs exhibit a bistable behavior. Indeed, multiple stable solution branches may exist for the same external parameters, so that the route taken to generate the comb determines its final state. In addition, changes to external pump intensity and detuning may change the MI gain and the dominant sideband pair, which leads to transitions between states with different number of pulses in the cavity.

Bichromatic pumping of a microresonator can be implemented by using two separate CW laser sources, with the beat note locked to a reference oscillator for high comb stability, or by modulating a single CW pump laser so as to simultaneously excite two cavity modes. This method leads to thresholdless (i.e., low power) FWM-induced comb generation, hence it could enable integrated chip-scale frequency comb sources. In this case the comb generation may again be described by a periodically (with frequency $\Omega$ ) driven and damped NLS equation [10]. With bichromatic pumping there is no CW steady-state (the pump field is not an equilibrium solution), which allows to generate background free patterns. The number of intracavity pulses can be controlled by the 
pump separation and is proportional to twice the modulation frequency $\Omega$.

A dual-pumped configuration allows the generation of combs where no soft excitations exist within the three-wave model, i.e., in the normal dispersion regime. Combs with a background $\mathrm{CW}$ component are generally found to be unstable in the anomalous dispersion regime, while they are stable for normal dispersion. The dual pumped configuration is also capable of supporting cavity solitons. These cavity solitons exist on a stationary pattern of low intensity pulses, and interactions between adjacent solitons are suppressed, which leads to a possible use of the microresonator as an optical bit buffer.

If the nonlinear development of MI in the anomalous dispersion regime of the cavity leads to a periodic train of pulses that connect the (stable) lower and (unstable) upper branches of the $\mathrm{CW}$ bistability curve, then a cavity soliton corresponds to isolating a single pulse from the train. An approximate description for cavity solitons may be provided by standard NLS soliton perturbation theory, by supposing that the loss and external injection are relatively weak [11]. Although in passive fiber cavities individual cavity solitons could be generated via cross-phase modulation by injecting an external writing pulse into the cavity [12], in microresonators it is possible to directly generate trains and even isolated cavity solitons by slowly sweeping the cavity detuning [13]. As a matter of fact, a stable and phase coherent frequency comb with a comb spacing equal to the cavity free-spectral range (FSR) is obtained with a single cavity soliton pulse circulating per round-trip.

\section{HIGH POWER REGIME}

Let us consider now frequency comb generation in the regime where the nonlinear length scale is comparable to the resonator length. Such a regime corresponds to nonlinear phase shifts of order unity, i.e., beyond the validity of the mean-field approximation. It is a common situation for fiber-ring resonators, but may also become important for microresonators in materials with large nonlinear coefficients (e.g. silicon nitride) at high pump powers. Large Kerr frequency mode shifts have in fact already been demonstrated experimentally, including nonlinear frequency shifts of up to $100 \mathrm{GHz}$ in silica microtoroids [21]. It may thus be expected that period doubling phenomena, multi-stability and additional instability mechanisms, previously observed in fiber cavities [14], should also be observable in microresonator frequency combs.

In these situations, the temporal dynamics should be analyzed by means of the Ikeda map directly [15]. The well known bistable CW solution of the LLE is very inaccurate at high intracavity powers, and it should therefore be replaced by the multi-valued stationary response of the map. Surprisingly, higher order stationary states of the map may also occur for similar pump powers as the usual bistable states.

The presence of periodic boundary conditions introduces additional instability mechanisms that are not found in the LLE, e.g., P2 instabilities and parametric instabilities similar to those found in dispersion oscillating fibers. A linear stability analysis of the steady state solutions of the map may therefore be used to determine growth rate of instabilities at a given modulation frequency. It is possible to find an analytical solution for the growth rate of unstable cavity modes if absorption losses are neglected. In the general case, the instabilities may be studied numerically by using Floquet theory. Besides ordinary MI, additional resonance instability mechanisms appear at high powers, owing to the periodic boundary conditions, both for anomalous and normal dispersion [15]. The different instability bands may be classified as either P2-instabilities, or ordinary MI. Typically the P2-MI is the first instability to occur for normal dispersion.

Quite remarkably, the Ikeda map also exhibits novel localized soliton states that occur due to the presence of multistability. Indeed, the cavity soliton regime is similarly associated with the presence of bistability in the LLE. For example, in the case where three $\mathrm{CW}$ levels are present simultaneously, a novel type of super cavity soliton exists, which is associated with the upper or excited CW state, in coexistence (i.e., with exactly the same pump and cavity parameters) with the LLE cavity soliton. These super cavity solitons are more energetic, and have a broader spectral bandwidth than LLE solitons, which is an intriguing property for wideband comb generation.

\section{APPLICATIONS}

The microring resonance spectrum and its dispersion properties must be engineered for particular applications, e.g., octave spanning comb generation. For wideband comb generation, one should preferably have anomalous cavity dispersion with a wide and flat dispersion profile [17][18]. Tailoring the linear dispersion and effective area properties of silicon microrings for efficient MIR frequency comb generation involves a suitable design of the microresonator radius and cross section. Indeed, a host of potential applications, including biomedical and environmental spectroscopy, arise in the MIR range of the spectrum. The natural candidate for implementing a chip-scale, MIR frequency comb source is silicon, because of its $10^{2}$ higher nonlinearity compared to silica, coupled with vanishing TPA and associated FCA and free-carrier dispersion (FCD) for photon energies below the silicon half band-gap. Recent experiments have demonstrated that silicon-chip based MIR frequency comb generation is critically dependent upon the possibility of reducing the lifetime of free carriers generated by three-photon absorption (3PA) [16].

Time dynamics of frequency comb generation in silicon microresonators is described by a generalized nonlinear envelope equation for the field envelope which includes linear loss and dispersion, the Kerr effect, Raman scattering, TPA and 3PA, FCA and FCD, coupled with the slow evolution equation for the cavity-averaged carrier density [17].

When pumping the TM mode, the parallel Raman gain vanishes in silicon: but the temporal evolution of the intracavity intensity shows that even for no pump detuning from cavity resonance, a stable soliton pattern is still generated from MI. Indeed, FCD associated with 3PA dynamically provides the nonlinear detuning which is necessary to sustain soliton propagation [17]. In order to arrive to a stable multi- 
soliton comb, it is necessary to properly adjust the free-carrier lifetime (FCT), which can be achieved by tuning the reverse bias voltage applied to a PIN structure embedding the microring [16]. For the formation of a stable soliton comb, the FCT should be comprised between a lower and an upper bound. As the FCT grows larger than a certain critical value, a Hopf bifurcation into a breathing comb is observed: the corresponding intracavity energy exhibits periodic explosions. Moreover, soliton annihilation resulting from collisions has been found to be accompanied by the simultaneous soliton creation at a different temporal position, so that the total soliton number is conserved, in order to keep the total FCD-induced detuning unchanged.

When pumping the TE mode, the presence of Raman gain dramatically changes the nature of the generated frequency comb. To maximize Raman gain, one should ensure that the peak of the silicon Raman gain at $15.6 \mathrm{THz}$ shift from the pump occurs for an integer multiple of the cavity FSR. In this case a Raman frequency comb is generated, featuring cascaded Raman Stokes lines of nearly equal intensity as the pump wave, as well as anti-Stokes comb lines, leading to octavespanning Raman comb bandwidths in excess of $100 \mathrm{THz}$ [17]. The associated temporal profile shows that the Raman comb results into the generation of a pulse train with about $15 \mathrm{fs}$ duration. Note that, because of the large Raman gain $\left(10^{4}\right.$ times the silica value) in silicon, stimulated Raman scattering remains the main comb generation mechanism also if the Raman gain shift is not precisely equal to a multiple of the FSR.

\section{REFERENCES}

[1] T. J. Kippenberg, R. Holzwarth, and S. A. Diddams, "MicroresonatorBased Optical Frequency Combs," Science, vol. 332, pp. 555-559, April 2011.

[2] K. Ikeda, "Multiple-valued stationary state and its instability of the transmitted light by a ring cavity system," Optics Communications, vol. 30, pp. 257-261, August 1979.

[3] Y. K. Chembo and N. Yu, "Modal expansion approach to opticalfrequency-comb generation with monolithic whispering-gallery-mode resonators," Physical Review A, vol. 82, pp. 033801, September 2010.

[4] A. B. Matsko, A. A. Savchenkov, W. Liang, V. S. Ilchenko, D. Seidel, and L. Maleki, "Mode-locked Kerr frequency combs," Optics Letters vol. 36, pp. 2845-2847, August 2011.

[5] S. Coen, H. G. Randle, T. Sylvestre, and M. Erkintalo, "Modeling of octave-spanning Kerr frequency combs using a generalized mean-field Lugiato-Lefever model," Optics Letters, vol. 38, pp. 37-39, January 2013.
[6] L. A. Lugiato and R. Lefever, "Spatial dissipative structures in passive optical systems," Physical Review Letters, vol.58, pp. 2209-2211, May 1987.

[7] M. Haelterman, S. Trillo, and S. Wabnitz, "Dissipative modulation instability in a nonlinear dispersive ring cavity,"' Optics Communications, vol. 91, pp. 401-407, August 1992.

[8] M. Haelterman, S. Trillo, and S. Wabnitz, "Additive-modulationinstability ring laser in the normal dispersion regime of a fiber," Optics Letters, vol.17, pp. 745-747, May 1992.

[9] T. Hansson, D. Modotto, and S. Wabnitz, "Dynamics of the modulational instability in microresonator frequency combs," Physical Review A, vol. 88, p. 023819, August 2013.

[10] T. Hansson and S. Wabnitz, "Bichromatically pumped microresonator frequency combs", Phys. Rev. A, vol. 90, p. 013811, July 2014.

[11] S. Wabnitz, "Suppression of interactions in a phase-locked soliton optical memory," Opt. Lett., vol. 8, pp. 601-603, April 1993.

[12] F. Leo, S. Coen, P. Kockaert, S.-P. Gorza, P. Emplit, and M. Haelterman, "Temporal cavity solitons in one-dimensional Kerr media as bits in an all-optical buffer," Nature Photonics, vol. 4, pp. 471-476 May 2010.

[13] T. Herr, V. Brasch, J.D. Jost, C.Y. Wang, N.M. Kondratiev, M.L. Gorodetsky, and T.J. Kippenberg, "Temporal solitons in optical microresonators," Nature Photonics, vol. 8, 145-152, February 2014.

[14] S. Coen and M. Haelterman, "Modulational instability induced by cavity boundary conditions in a normally dispersive optical fiber," Physical Review Letters, vol. 79, pp. 4139-4142, November 1997.

[15] T. Hansson and S. Wabnitz, "Frequency comb generation beyond the Lugiato-Lefever equation: multi-stability and super-cavity solitons," J. Opt. Soc. Am. B vol. 32, pp. 1259-1266, July 2015.

[16] A. G. Griffith, R. K. W. Lau, J. Cardenas, Y. Okawachi, A. Mohanty, R. Fain, Y. H. D. Lee, M. Yu, C. T. Phare, C. B. Poitras, A. L. Gaeta, and M. Lipson, "Silicon-chip mid-infrared frequency comb generation," Nature Communications, vol. 6, p. 6299, February 2015.

[17] T. Hansson, D. Modotto and S. Wabnitz, "Mid-infrared soliton and Raman frequency comb generation in silicon microrings," Opt. Lett., vol. 39, pp. 6747-6750, December 2014.

[18] Y. Okawachi, M. R. E. Lamont, K. Luke, D. O. Carvalho, M. Yu, M. Lipson and A. L. Gaeta, "Bandwidth shaping of microresonator based frequency combs via dispersion engineering", Opt. Lett., vol. 39, pp. 3535-3538, June 2014.

[19] T. Hansson, D. Modotto, and S. Wabnitz, "Analytical approach to the design of microring resonators for nonlinear four-wave mixing applications," J. Opt. Soc. Am. B, vol. 31, pp. 1109-1117, May 2014.

[20] T. Hansson, D. Modotto, and S. Wabnitz, "On the numerical simulation of Kerr frequency combs using coupled mode equations," Optics Communications, vol. 312, pp. 134-136, February 2014.

[21] P. Del'Haye, A. Coillet, W. Loh, K. Beha, S. B. Papp, and S. A. Diddams, "Phase steps and resonator detuning measurements in microresonator frequency combs," Nature Communications vol. 6, p. 5668, February 2015. 